\title{
CORRELATED ERROR ANALYSIS FOR THE NON-LINEAR OPTIMIZATION AOA GEOLOCATION ALGORITHM
}

\author{
Joshua Sprang, Derek Hesser, Jason Roos, Jonathan Mautz, \\ Matthew Sambora, Clark Taylor, Joseph Sugrue, Andrew Terzuoli \\ Institute of Electrical \& Electronic Engineers (IEEE), Dayton, Ohio USA
}

a.j.terzuoli@ieee.org

\begin{abstract}
Previously the Gauss Newton method has been used to estimate the geo-location of an object from angle of arrival (AoA) measurements. This method has assumed, however, that all measurements were independent and identically distributed. Real sensor data, however, often has temporal correlations between measurements. If a detailed understanding of the measurement correlation exists, this correlation can be explicitly modeled and jointly estimated with the geo-location. Obtaining a detailed and accurate model of measurement error correlation, however, is often infeasible for a system where the unit producing measurements may be a black box. To overcome this unknown correlation between measurements, we propose a modified Gauss-Newton optimization algorithm based on prior Covariance Intersection work. A discussion on the efficacy of this modified technique, in terms of both geolocation accuracy and accurate prediction of geo-location uncertainty, concludes the paper.
\end{abstract}

Index Terms - Non-Linear Optimization, Covariance Intersection, Angle of Arrival, Error Depiction, Location Estimation

\section{INTRODUCTION}

An important application for remote sensing involves estimating the geolocation of an object as well as the uncertainty of the given estimates. To produce geo-location estimates, this paper will focus on angle-of-arrival measurements from remote sensors. AoA can be derived from a variety of sensors ranging from electro-optical imagers to RADAR systems. In previous work, a non-linear optimization (NLO) algorithm based on a generalized Gauss-Newton algorithm is used to produce optimal estimates from the AoA measurements [1][2]. With the nonlinear optimization algorithm the estimation of an object's state occurs simultaneously to the estimation of a covariance matrix that represents the uncertainty in the estimated geo- location. Calculating the uncertainty is important for measuring the ability of a system of sensors to locate an object.

While the approach in [1][2] yields optimal estimates, the proposed algorithm assumes that every measurement has independent and identically distributed (IID) additive noise, and that the uncertainty of that noise is known a-priori. Unfortunately, this assumption is often violated with real-world sensors. While the uncertainty in a measurement is not infrequently known for a wellcharacterized sensor, the time-correlation between measurements is rarely well-characterized. For example, consider a typical GPS receiver. Most GPS receivers will return a location estimate together with the uncertainty associated with that estimate. The location estimates over time, however, are not independent. The correlation between location estimates though is not typically produced by the GPS receiver (the authors are not aware of any GPS receivers that produce time correlation outputs!)

A time-correlated measurement noise (bias) can arise from a number of factors. To handle these biases, they can either be measured a-priori, or possibly estimated as another value in the state estimate if the characteristics of the biases are known. However, in many cases a sensor's individual bias will be unknown, is difficult to measure apriori, and adding it to the estimation state may expand the state past a point of estimation stability (particularly when the time correlation constant is relatively small) or otherwise negatively affect the overall estimation accuracy.

In this paper, we introduce a novel way to handle the presence of biases within the optimization algorithms used for triangulating object locations. Using Covariance Intersection [3] based ideas, the non-linear optimization algorithm is modified to consider the presence of time correlation between measurement inputs, with the assumption that only measurements from the same sensor are correlated (i.e. only time-correlation is present, not cross-sensor correlation.) In the remainder of this paper, we present the modified optimization algorithm, an 
environment for evaluating this algorithm, and results demonstrating the efficacy of this approach.

\section{ALGORITHM}

Covariance Intersection as defined by Julier and Uhlmann [3][4], is used to fuse data together from disparate sources when there is an unknown amount of correlation between the data sources. Traditional data fusion, where all measurements are uncorrelated, can be written as:

$$
\begin{aligned}
& \Sigma_{c}{ }^{-1}=\sum_{n=1}^{N} \Sigma_{m, n}{ }^{-1}, \\
& \bar{c}=\Sigma_{c} \sum_{n=1}^{N} \Sigma_{m, n}{ }^{-1} \bar{m}_{n},
\end{aligned}
$$

where $\Sigma_{m, n}$ represents the covariance matrix where the $\mathrm{m}$ subscript stands for measurement and $\mathrm{n}$ is the measurement index. The total number of measurements is $\mathrm{N}$. To handle the presence of unknown correlations between the different measurements, Covariance Intersection add a weight $(\omega)$ to the above summations as follows:

$$
\begin{aligned}
& \Sigma_{c}^{-1}=\sum_{n=1}^{N} \omega_{n} \Sigma_{m n}^{-1}, \\
& \bar{c}=\Sigma_{c} \sum_{n=1}^{N} \omega_{n} \Sigma_{m n}{ }^{-1} \bar{m}_{n}, \\
& \sum_{n=1}^{N} \omega_{n}=1 .
\end{aligned}
$$

If some information about the correlation between measurements is known, the constraint of all the weights summating to 1 can be relaxed somewhat [4], but in general, the correlated portion of the measurements is handled by insuring the summation of all the weights is equal to 1 .

To apply Covariance Intersection to the non-linear optimization problem described in [1][2], we modified the formula (1) to weight all measurements from a single sensor equally. We also assume that there is no correlation between different sensors; we are using Covariance Intersection to compensate for temporal, but not cross-sensor correlations. Assuming that the correlation of error is unknown for multiple measurements from a single sensor, and multiple sensors are being used for a single geolocation estimate, the non-linear optimization algorithm becomes (for two sensors)

$$
\begin{aligned}
& \Sigma_{c}^{-1}=\sum_{n=1}^{N} \omega_{n} \Sigma_{m n}{ }^{-1}+\sum_{k=1}^{K} \omega_{k} \Sigma_{m k}{ }^{-1}, \\
& \sum_{n=1}^{N} \omega_{n}=1, \\
& \sum_{k=1}^{K} \omega_{k}=1 .
\end{aligned}
$$

with the algorithm trivially extended to multiple sensors. (The variables $N$ and $K$ represent the number of measurements from two different sensors.)

For the non-linear optimization algorithm, the covariance matrix $\Sigma_{X}$ depicting the error in an estimated state is calculated using the Jacobian matrix $\mathbf{J}$ and the covariance matrix of the sensor measurements $\Sigma_{m n}$ via

$$
\Sigma_{X}{ }^{-1}=\sum_{n=1}^{N} \mathbf{J}_{n}^{\top} \Sigma_{m n}^{-1} \mathbf{J}_{n}
$$

Using Covariance Intersection, the state estimate's covariance matrix using two different sensors is

$$
\Sigma_{X}{ }^{-1}=\sum_{n=1}^{N} \omega_{n}\left(\mathbf{J}_{n}^{\top} \Sigma_{m n}{ }^{-1} \mathbf{J}_{n}\right)+\sum_{k=1}^{K} \omega_{k}\left(\mathbf{J}_{m}{ }^{\top} \Sigma_{m k}{ }^{-1} \mathbf{J}_{k}\right) .
$$

It is possible to calculate the state estimate's covariance matrix with more than two sensors by following the additive pattern above. The covariance matrix is used within the non-linear optimization algorithm via

$$
\Delta \mathbf{X}=\Sigma_{X}\left(\sum_{n=1}^{N} \omega_{n} \mathbf{J}_{n}^{\top} \Sigma_{m n}^{-1} \Delta \Omega_{n}+\sum_{k=1}^{K} \omega_{k} \mathbf{J}_{m}^{\top} \Sigma_{m k}{ }^{-1} \Delta \Omega_{n}\right)
$$

The non-linear optimization using Covariance Intersection will produce larger error ellipses than without Covariance Intersection when depicting error. The more measurements from a single sensor that are used, the lower the weights become for each covariance matrix due to the correlation among measurements. In the results, we show that this increase in estimated uncertainty more accurately represents the true uncertainty than the IID, assumption utilized in the traditional non-linear optimization approach. In addition to being more accurate, note that no information about the time-correlation (e.g., cause of bias, time constant for the correlation, dynamic model, etc.) is required, making 
it unnecessary to have a detailed knowledge of the noise source and model it within the estimation framework. Therefore, the proposed approach is both considerably simpler and more accurate than other approaches to handling time-correlation!

\section{EVALUATION}

The evaluation of the algorithm was performed on a scenario representing the triangulation of an object of interest from satellites in space. This includes setting up a simulated path for an object of interest and a simulation of measurements from a group of sensors tracking the object. Object paths are simulated based on orbital mechanics. The objects are created with circular orbits around the earth at different low earth altitudes. The speed of the object is dependent on the object's altitude in order to simulate a realistic system. Sensor positions are randomly generated in space such that the earth cannot interfere with the line of sight needed to produce the AOA measurements. The altitudes are used to calculate the orbital periods of sensors. Random jitter is created with the assumption that it follows a normal distribution. The added bias is created by applying two cumulative sums on uniform random noise. A uniform distribution is chosen to simulate the truncating of a voltage on an analog to digital converter being used to read an accelerometer. A double accumulation of error simulates the noise on the acceleration readings being converted to positional noise. The variances for the single sensors take into account the random jitter and the bias being produced. To evaluate the algorithm's effectiveness in producing error ellipses, multiple simulations are generated for a MonteCarlo experiment.

\section{ERROR ANALYSIS}

In the previous papers [1][2], the estimated uncertainty when using the traditional NLO was found to be consistent in simulation assuming the inputs were IID, but without time-correlated measurement noise. The metrics we used to define the accuracy of estimating uncertainty include normalized estimation error squared (NEES) and mean squared error (MSE), which are dependent on the amount of simulated time correlation measurement error. NEES can be defined as

$$
\mathrm{NEES}=\frac{1}{N} \sum_{i=1}^{N}\left(X_{i}-\hat{X_{i}}\right) \sum_{m n}^{-1}\left(X_{i}-\hat{X_{i}}\right)
$$

where $\mathrm{X}_{\mathrm{i}}$ is the true location and $\hat{X}_{i}$ is the estimated location. On the other hand, MSE is utilized as a reference to relate the traditional and enhanced NLO methods in order to provide a comparison alongside the well-known
Triangulation algorithm for geolocation estimation since Covariance Intersection was not applied to Triangulation.

NEES aids in describing the overall confidence of estimated uncertainty and the accuracy of the geolocation estimation produced from the NLO. For geolocation estimation purposes one is typically interested most in the position estimation from the object state. In 3-D space, position estimate has three degrees of freedom for each axis $\mathrm{x}, \mathrm{y}$, and $\mathrm{z}$. An NEES value of three implies that the calculation of uncertainty error in the system is an accurate representation of the error in the geolocation of the position compared to the true unknown location with respect to three standard deviations. An NEES value greater than three implies over-confidence in the uncertainty estimation. Overconfidence implies that the quantification of error for each estimate encompasses less error than intended, resulting in inaccurate estimations of uncertainty. Therefore, an underconfident estimation of uncertainty is desirable for the fact that additional error in the system is included. This allows for one to state a higher level of confidence in the accuracy of the geolocation estimation compared to the unknown truth.

Table 1 depicts the resulting metrics for analyzing the error analysis for 100 Monte-Carlo simulations with an averaged simulated bias of 2.6 milli-radians/second and 25 micro-radians/second of jitter of error added onto the AoA data. When introducing time-correlated measurement noise, the previous NLO is shown to provide an extremely high NEES value, implying a far too over-confident estimation of uncertainty error. However, upon introducing Covariance Intersection it can be seen that the NEES value decreases to below three, or the degrees of freedom for the geolocation estimate. These results draw the conclusion that without Covariance Intersection the presence of time-correlated measurement error degrades the previous optimization algorithm's ability to depict error. Once Covariance Intersection is applied to the NLO algorithm, higher levels of bias no longer cause the non-linear optimization to produce over-confident estimations. Also note that Covariance Intersection does not significantly decrease the accuracy of the estimates (denoted by an increase in MSE) despite being more consistent.

\begin{tabular}{|c|c|c|}
\hline & NEES & MSE \\
\hline Triangulation & N/A & $3.8325^{*} 10^{\wedge} 11$ \\
\hline NLO & 26.77 & $1.3721^{*} 10^{\wedge} 11$ \\
\hline $\begin{array}{c}\text { NLO with } \\
\text { Covariance } \\
\text { Intersection }\end{array}$ & 1.9862 & $1.4035^{*} 10^{\wedge} 11$ \\
\hline
\end{tabular}

Table 1 results and metrics to describe uncertainty estimation 


\section{CONCLUSION}

Sensor error is more accurately described using a bias in conjunction in addition to IID noise. Using Covariance Intersection to account for correlated measurements from a bias decreases the overall confidence in an estimated state, but is considerably better than ignoring the bias and is considerably simpler than many past methods accounting for bias. One possible drawback of covariance-intersection based techniques is that the algorithm may become underconfident. However, we believe that in many situations, under-confident error estimations are more desirable than over-confident estimates. Though true confidence is the overall goal, an under-confident estimation ensures with respect to three standard deviations that an object's location exists within the calculated error.

\section{REFERENCES}

[1] L. Burchett, S. Hartzell, G. Hoffar, J. Mautz, C. Taylor, and A. Terzuoli. "Angle of arrival geolocation using non-linear optimization”. 2012 IEEE International Geoscience and Remote Sensing Symposium (IGARSS), 1-4. 2012. ISSN 2153-6996.

[2] S. Hartzell, M. Haker, R. Martin, C. Taylor, and A. Terzuoli. "Angle of arrival geolocation for fast-movers using nonlinear optimization". 2014 IEEE International Geoscience and Remote Sensing Symposium (IGARSS), 1-4. 2014.

[3] S.J. Julier, J.K. Uhlmann, "A non-divergent estimation algorithm in the presence of unknown correlations," American Control Conference, 1997. 2369-2373. 1997.

[4] S.J. Julier, J.K. Uhlmann, "Simultaneous localisation and map building using split covariance intersection," 2001 IEEE/RSJ International Conference on Intelligent Robots and Systems. 12571262. 2001. 